\title{
Use of the Pill Electrode for Transesophageal Atrial Pacing
}

\author{
JANICE M. JENKINS, MACDONALD DICK, STEVE COLLINS,* \\ WILLIAM O'NEILL, ROBERT M. CAMPBELL, and DAVID J. WILBER \\ From the Departments of Electrical Engineering and Computer Science, Pediatrics, and Internal \\ Medicine, The University of Michigan, Ann Arbor, Michigan and *Departments of Electrical \\ and Computer Engineering and Radiology, The University of Iowa, Iowa City, Iowa
}

\begin{abstract}
JENKINS, J.M., ET AL.: Use of the pill electrode for transesophageal atrial pacing. The pill electrode, which was developed for esophageal electrocardiography, has found application in transesophageal atrial pacing during procedures such as conversion of tachycardia, electrophysiologic measurement, and acceleration of heart rate to produce stress during cardiac imaging studies. This paper presents theoretical studies that examine the relationship of interelectrode distance, current level, and pulse duration to the achievement of successful capture. Theoretical results agree with our clinical findings, i.e., current levels of $25 \mathrm{~mA}$ are effective to sustain capture; increased pulse duration reduces current requirements; and close bipolar spacing combines efficacy with safety. Results of animal studies performed to assess the extent of esophageal burn injury reveal that current levels in excess of $75 \mathrm{~mA}$ are required to produce lesions in short-term (under 30 minutes) pacing, and greater than $60 \mathrm{~mA}$ in long-term ( 4 hours) pacing. These results are based on experiments using a pulse duration of $2 \mathrm{~ms}$, and the current levels that produce injury will be considerably lower if longer pulse durations are used. Typical current levels and pulse durations for successful capture are presented for 46 subjects in several new clinical applications. Termination of tachycardia, basic electrophysiologic measurements, and controlled acceleration of heart rate can be performed noninvasively with this technique. (PACE, Vol. 8, July-August, 1985)
\end{abstract}

esophageal, atrial, pacing, noninvasive

\section{Introduction}

The recently developed pill electrode, widely used for esophageal electrocardiography, can also be employed for short-term transesophageal left atrial pacing. Thus, it is possible to perform a variety of procedures in which atrial pacing can be done noninvasively with minimal time and patient discomfort. These procedures include: initiation and termination of tachycardia, delivery of single and multiple extrastimuli for the measurement of electrophysiologic and hemodynamic

Supported in part by NIH Biomedical Research Support Grant to the Vice President for Research at The University of Michigan, American Heart Association, Iowa affiliate grant 78-6-2, and NIH grant 1R43HL32378-01.

Address for reprints: Dr. Janice M. Jenkins, Dept. of Electrical \& Computer Engineering, The University of Michigan, Ann Arbor, MI 48109.

Received September 6, 1984; revision received February 19, 1985; accepted March 11, 1985. response, and acceleration of heart rate to produce stress during imaging studies of ventricular function. In this last application, transesophageal pacing can provide more precise control of heart rate during echocardiographic, radiographic, or nuclear imaging than can be obtained with exercise; it also eliminates image degradation due to patient movement. These applications prompted us to study the theoretical basis of transesophageal atrial pacing, its safety in animals, and some new clinical applications.

\section{Background}

Several groups interested in atrial pacing have sought to avoid the discomfort, time, and cost of venous catheterization by introducing the pacing electrode through the nose or mouth down into the esophagus to the level of the heart. Early efforts to pace the heart ${ }^{1-4}$ were limited for one of four reasons: (1) the studies were done in dogs, whose thoracic geometry is such that the esoph- 
ageal-cardiac approximation is poor; (2) the effort concentrated on pacing the human ventricle, which is not particularly close to the esophagus; (3) unipolar electrodes were used and the "indifferent" electrode caused undesirable stimulation of chest and diaphragmatic musculature; and (4) the stimulator employed did not deliver enough current.

Later studies concentrated on atrial pacing in humans using bipolar electrodes and high-voltage stimulators, ${ }^{5-17}$ and had successful left atrial capture at currents between 10 and $30 \mathrm{~mA}$ with rare ventricular or chest muscle stimulation. These studies and our own preliminary studies ${ }^{18,19} \mathrm{dem}$ onstrate the technical feasibility of transesophageal pacing and suggest that broader application may develop quickly. Nevertheless, there are three important aspects of transesophageal pacing that have not been adequately addressed and that are important to its future usefulness as a diagnostic and therapeutic technique: (1) the physical and mathematical principles underlying the method have not been presented, so that users may lack a clear understanding of several important parameters of the technique, including choice of bipolar spacing, effect of electrode position, and current and voltage requirements; (2) how the discomfort of nasogastric intubation may limit the acceptability of the technique; and (3) the lack of animal studies done to assess the risk of electrical burning of the esophagus. The present study was designed to address these deficiencies.

\section{Theoretical Principles}

The current density required to pace the heart from electrodes in proximity to excitable tissue can be estimated by first considering a configuration that is known to be effective and that is easily described mathematically, i.e., a conducting ring on a catheter in contact with the endocardium, as shown in Figure 1. For such an electrode, the current density at its surface is given by the electrode current divided by the electrode area

$$
\mathrm{J}=\frac{\mathrm{I}}{\pi \mathrm{dl}}
$$

where $\mathrm{I}$ is the current, $\mathrm{d}$ and $\mathrm{l}$ are the electrode diameter and length, and $J$ is the current density at the electrode surface. Equation 1 is exact in a homogeneous medium for a cylinder that is long compared to its radius. In our case, the lengthto-radius ratio is $4: 1$ and the endocardium and intracavitary blood have essentially the same resistivity, so the equation provides a reasonable first approximation. For a typical catheter elec-

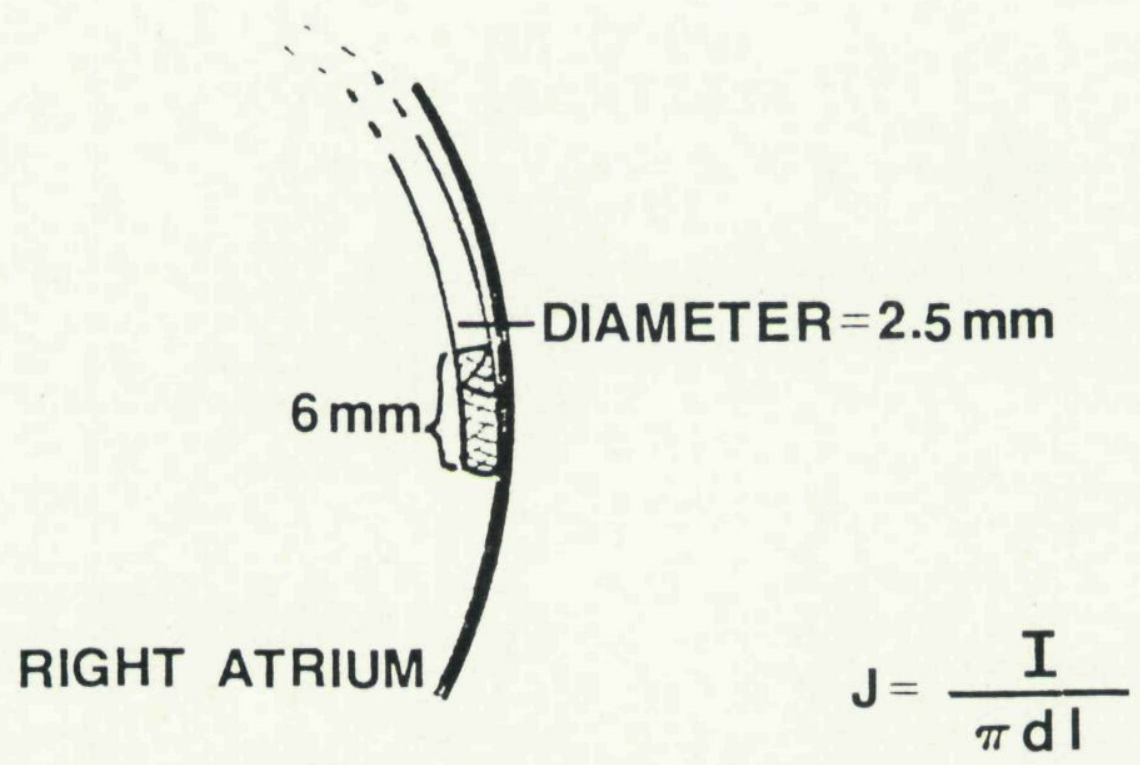

Figure 1. Drawing of a cylindrical catheter electrode in contact with the endocardial surface of the right atrium. Current density $(J)$ present in adjacent tissue is determined by the amplitude of current (I) delivered, divided by area of the electrode surface. ( $d=$ diameter, $1=$ length) 
trode, these numbers are $\mathrm{I}=1 \mathrm{~mA}, \mathrm{~d}=2.5 \mathrm{~mm}$, $\mathrm{l}=6 \mathrm{~mm}$. The resulting current density, $20 \mu \mathrm{A} /$ $\mathrm{mm}^{2}$, provides a fairly accurate estimate of the current density actually present in endocardial tissue during catheter pacing. The $1 \mathrm{~mA}$ current would be adequate for a stimulation pulse of 1$2 \mathrm{~ms}$, which is typical in catheter pacing. (Inhomogeneity and boundary effects, which complicate such calculation in the usual thoracic volume conductor studies of theoretical electrocardiology, are of little significance in this case, since the electrode is close to the structure being stimulated.)

The esophageal case is shown in Figure 2. We postulate a $10 \mathrm{~mm}$ distance from esophagus to left atrium and assume the same $1 \mathrm{~mA}$ current source delivered to a small spherical unipolar esophageal electrode in a homogeneous medium. The current density is

$$
J=\frac{I}{4 \pi R^{2}}=\frac{1}{4 \pi(10)^{2}}=0.8 \mu \mathrm{A} / \mathrm{mm}^{2}
$$

where $\mathrm{R}$ is the distance in millimeters from the center of the small electrode to excitable tissue. Similarly, for a bipolar esophageal electrode located at the same distance from the left atrium and with $13 \mathrm{~mm}$ interelectrode spacing (Fig. 3), it can be shown that

$$
\begin{array}{r}
J=\frac{\text { bI }}{4 \pi\left[\mathrm{R}^{2}+\left(\frac{\mathrm{b}}{2}\right)^{2}\right]^{3 / 2}}=\frac{(13)(1)}{4 \pi(142.3)^{3 / 2}} \\
=0.61 \mu \mathrm{A} / \mathrm{mm}^{2}
\end{array}
$$

where $\mathrm{b}$ is the bipolar electrode spacing and $\mathrm{R}$ is the distance from the midpoint of the electrode to the nearest excitable tissue on the midplane of the electrode. Comparison of equations 1, 2,

\section{ESOPHAGUS}

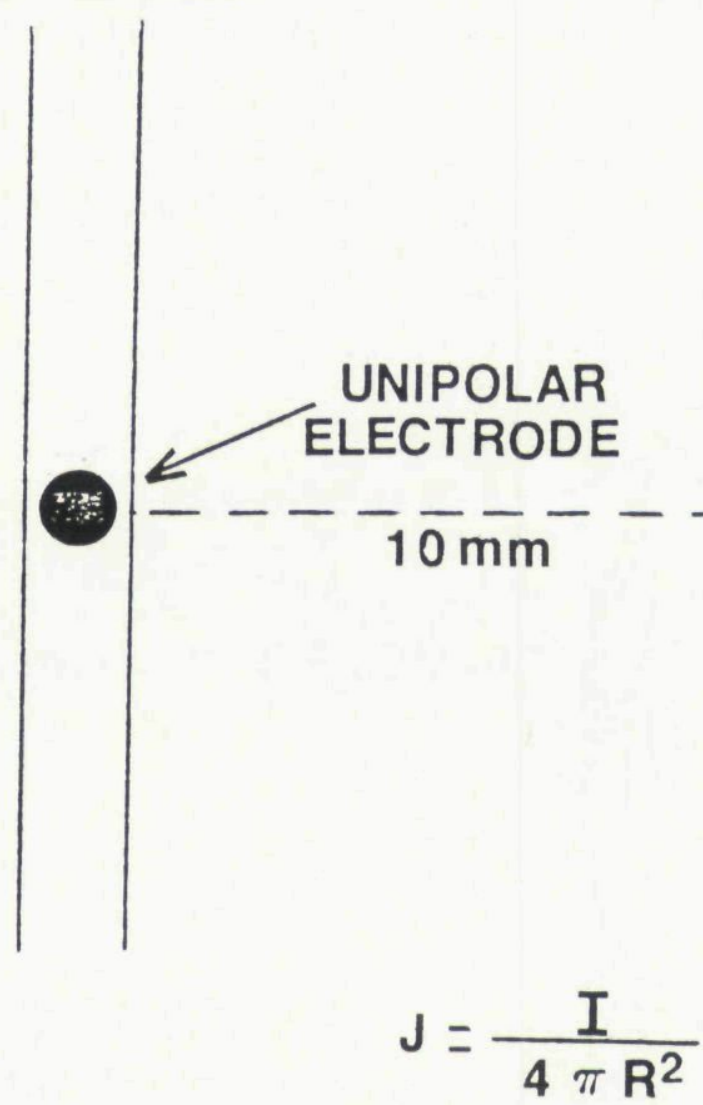

Figure 2. Schematic of unipolar pacing electrode within the esophagus shown with estimated distance from nearest atrial surface. Current density $(J)$ within the left atrium is inversely related to the square of the distance $(\mathrm{R})$ from electrode to atrial excitable tissue. 


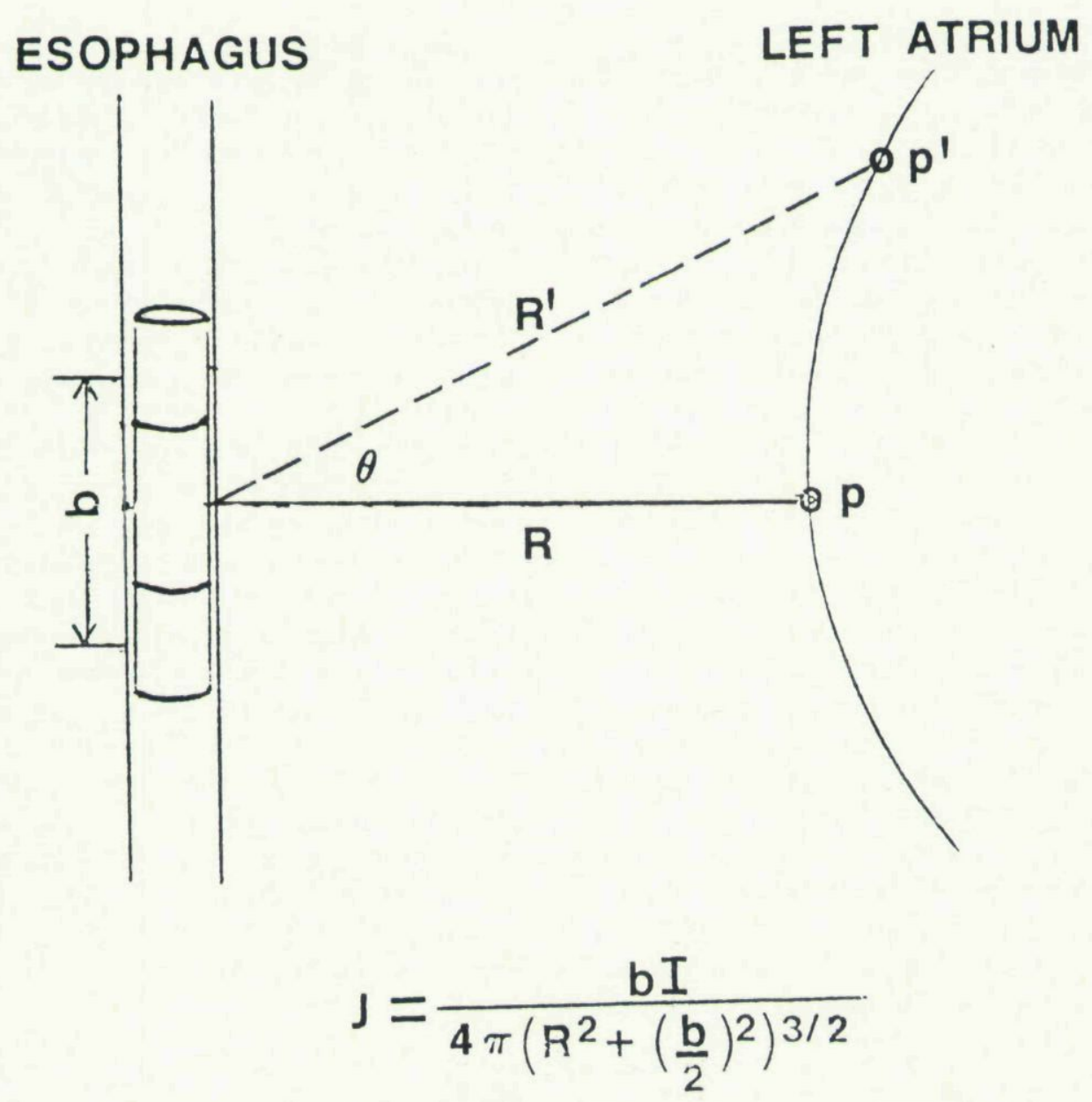

Figure 3. Bipolar esophageal electrode showing relationship of current density (J) to interelectrode spacing (b) and distance from excitable tissue (R). Point p represents closest atrial myocardium on the midplane of electrode. If electrode is positioned such that closest atrial tissue is not located on bipolar midplane $\left(\mathrm{p}^{\prime}\right)$, the angle of displacement $\theta$ is a factor in the current density calculation. See equation 4 in text. ( $I=$ current $)$

and 3 indicates that the current density delivered to the atrium by an esophageal electrode is about $1 / 25$ that from an intracavitary lead. In addition, the current density from the unipolar electrode is perpendicular to the tissue, whereas that from the bipole would be tangential to the left atrium. It may be that current densities perpendicular to fibers are more effective in stimulating the tissue. On the other hand, atrial muscle does not have preferential direction, and so even the tangential current density will be perpendicular to many atrial fibers. Source currents of up to $25 \mathrm{~mA}$ appear to be necessary in an esophageal electrode to produce a cardiac current density sufficent to pace the heart. (Our preliminary experimental results in over 40 patients and volunteers have confirmed the theoretical conclusion that $25 \mathrm{~mA}$ is required to capture the atrium with a pulse duration of $1 \mathrm{~ms}$; wider pulses reduce the required current.)

Equation 3 can also be used to derive an optimal relationship between the bipolar spacing $\mathrm{b}$ and distance from the electrode $\mathrm{R}$ to obtain maximum current density when the electrode is properly positioned within the esophagus as close as possible to the left atrium. That relationship is $\mathrm{b}=\sqrt{2} \mathrm{R}=1.4 \mathrm{R}$. Thus the interelectrode spacing can be optimized for particular anatomical cases. In infants, for example, left atrial myocardium may be within $5 \mathrm{~mm}$ of the esoph- 
agus, and a bipolar spacing of $7 \mathrm{~mm}$ would produce the theoretically maximum current density at the atrium. Note that for distances in the usual adult range $(10 \mathrm{~mm})$, a bipolar spacing of $14 \mathrm{~mm}$ is optimal for pacing the left atrium with minimal current, reducing the risk of accidental ventricular stimulation and producing the least discomfort. We have found that for spacings within $5 \mathrm{~mm}$ of optimal, the current density at the atrial distance is within $10 \%$ of the maximum. For larger spacings ( $30 \mathrm{~mm}$ or more), the current density decreases sharply at the electrode midplane. For this case, capture will be more easily achieved if the bipolar electrode is moved from a symmetric position relative to the atrium to a position where either pole is adjacent to the excitable tissue and the other is remote. This situation essentially represents unipolar pacing and is likely to result in inadvertent stimulation of other structures.

Cardiac locations above or below the bipolar midplane of Figure 3 suffer a further loss of current density not shown in equation 3 . The general expression for this case is too complex to provide physiologic insight, but the following approximation exists for the current density when the distance from the electrode is large compared to the bipolar spacing:

$$
J=\frac{b I}{4 \pi R^{\prime 3}} \sqrt{4 \sin ^{2} \theta+\cos ^{2} \theta}
$$

where $\theta$ is the angle above the electrode midplane (Fig. 3) and the other symbols are as previously defined. Equation 4 reveals the important fact that when the distance from the electrode to the heart is much greater than the interelectrode spacing, the current density falls off inversely with the cube of distance, thus explaining why bipolar pacing minimizes inadvertent stimulation of remote structures. Even in the case where the distance is not much greater than the interelectrode spacing, the current density falls off dramatically. For example, in adults, the nearest ventricular surface is at least $3 \mathrm{~cm}$ from the esophagus, which is three times the distance of the posterior left atrial wall from the esophagus. For this case, detailed calculations from equation 4 reveal that a closely spaced bipolar esophageal electrode positioned for atrial pacing would produce ventricular current densities with only $\frac{1}{15}$ the strength of those produced in the left atrium.
Similarly, pectoral and diaphragmatic musculature are relatively insensitive to properly positioned bipolar transesophageal pacing.

Unfortunately, these proximity effects that are well known for closely spaced bipolar electrodes and are described concisely in equation 4 present a requirement for very careful positioning, as compared to unipolar or wide bipolar configurations. Loss of atrial capture can occur when a bipolar pacing electrode moves only a few centimeters.

We have also considered an esophageal/chest bipolar electrode combination for transthoracic pacing. From the viewpoint of the left atrium, such a configuration is essentially unipolar and has no advantages. Current levels of about 25 $\mathrm{mA}$ are painful to the skin and produce uncomfortable excitation of skeletal muscles. The esophageal mucosa is much less sensitive and only minor discomfort is experienced with bipolar pacing at $25 \mathrm{~mA}$. On the other hand, if ventricular pacing is intended, the distance of the ventricle from the esophagus averages $4 \mathrm{~cm}$ and unipolar electrodes, both thoracic and esophageal, will require less current than bipolar esophageal electrodes for equivalent ventricular current densities. ${ }^{20}$

\section{Methods}

\section{The Pill Electrode}

The pill electrode, ${ }^{*}$ which was designed originally for esophageal recording of the atrial electrogram, ${ }^{21,22}$ is shown in Figure 4. It consists of two conducting members whose centers are 13 $\mathrm{mm}$ apart, and thus it is theoretically optimal for pacing tissues at a distance of $9.4 \mathrm{~mm}$ from the esophagus. The electrode is attached to a twisted pair of very thin threadlike, Teflon-insulated, stainless steel wires. It is enclosed in an ordinary pharmaceutical gelatin capsule. The patient is instructed to swallow the capsule with water while the nurse or technician gradually releases the wire and allows the capsule to descend. Within a few moments the capsule dissolves and peristalsis has lowered the electrode to a level below the left atrium (approximately $40 \mathrm{~cm}$ from the teeth). At this time, the wire pair is connected

\footnotetext{
* Arzco Medical Electronics, Inc., Chicago, IL, USA.
} 


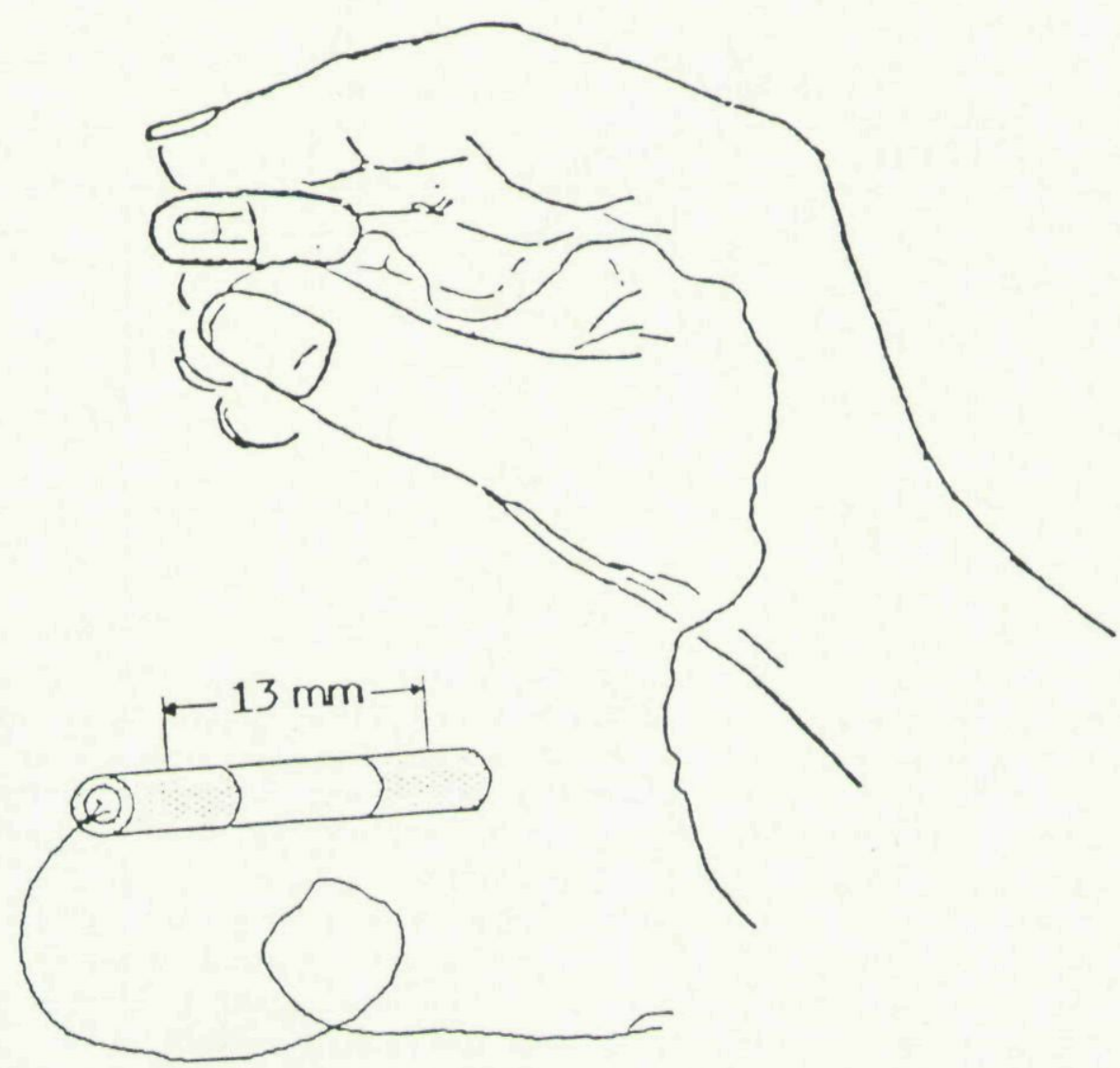

Figure 4. Drawing of the swallowable esophageal electrode encased in a gelatin capsule. The pill electrode is a closely spaced bipolar pair, suspended from a pair of thin flexible wires. The electrode passes into the eosphagus with a swallow of water followed by additional swallowing until it is positioned immediately posterior to the atrium.

to an electrocardiograph and slowly withdrawn a few centimeters at a time until the electrode is at an optimal level for atrial pacing. This level is indicated by a P-to-QRS ratio of 2 or 3 to 1 on the electrocardiogram (this ratio is an excellent indicator of left atrial approximation; radiography is unnecessary).

Figure 5 is an esophageal recording showing the $\mathrm{P}$-wave progressively emerging as the electrode is gradually raised from a low ventricular to a high atrial position. The atrial spikes are negative at first and increasing in amplitude. Then, there is a sudden change in polarity. This is the position where the nearest atrial myocardium is on the midplane of the electrode. When the electrode is properly positioned, the wire is taped to the patient's chin to prevent further peristaltic lowering and its connector is removed from the electrocardiograph and connected to the stimulator to begin pacing. Throughout this procedure, there is little gagging or consciousness of the fine flexible wire, so that the swallowing, recording, and positioning procedure is virtually innocuous.

\section{Stimulator}

The stimulator used for transesophageal atrial pacing must possess certain unique characteristics. As described in the theoretical section of this paper, the currents typically required for consistent capture range from 15 to $25 \mathrm{~mA}$. We measured the voltage during pacing at various currents and found that it is not strictly proportional to current delivered. Voltage also varies during swallowing, respiration, and changes in posture. If one defines the electrode impedance to be the ratio of voltage to current of the pacing 


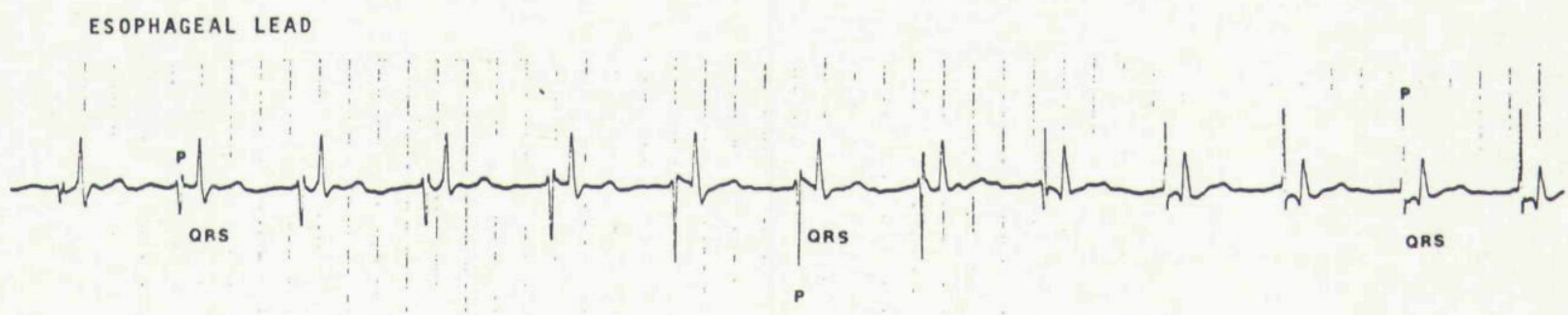

LEAD II

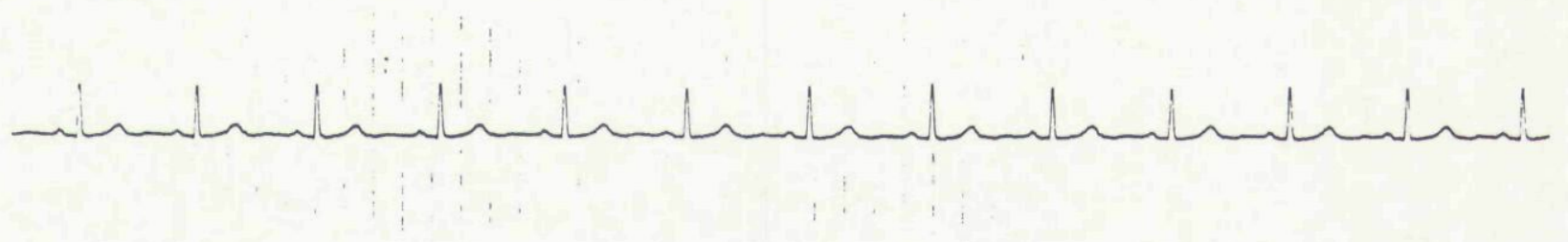

Figure 5. Two-channel tracing showing esophageal lead during positioning. The top channel is the esophageal electrogram and the lower channel a surface lead II. The esophageal lead is being withdrawn from below the ventricle (far left portion of tracing) to a position immediately posterior to the left atrium (far right portion of tracing). Note the progressively emerging Pwave which, when optimally located, shows a 2.5:1 ratio with respect to the esophageal QRS.

pulse, the impedance of the pill electrode ranges from 1,000 to $2,000 \mathrm{ohms}$ or more probably due to uncertain contact with the esophageal wall with its higher resistivity. Since atrial capture depends on current density in the excitable tissue, not voltage at the electrodes, the stimulator must provide a constant current over a wide range of impedances. It can be seen that the output voltage developed by the device can rise to 75 volts or more; therefore existing stimulators designed for endocardial pacing at impedances significantly less than $1,000 \mathrm{ohms}$ will not have the voltage capability to drive an esophageal electrode at 15 to $25 \mathrm{~mA}$ regardless of the rated current capability of the unit. Existing stimulators are typically battery-operated with a voltage limitation of 9 , 18 , or, at the most, 27 volts.

It is a well-known physiological observation that the pulse duration necessary to generate a propagated action potential in remote tissue is much longer than the 0.5 to $1 \mathrm{~ms}$ stimulus duration required when the electrode is in contact with the excitable tissue. Distances of about 10 $\mathrm{mm}$ exist between the esophagus and nearest excitable atrial tissue and thus wide stimulus pulses will be required. Our experimental findings which will be discussed in the next section demonstrate that the threshold of stimulus strength required for capture approaches the rheobase $^{23}$ as the pulse duration approaches 10 ms. In most of our experiments we have used a stimulator designed specifically for esophageal pacing (Arzco Medical Electronics) that can provide a constant current pulse of 0 to $40 \mathrm{~mA}$ and up to $10 \mathrm{~ms}$ width at pacing rates from 30 to 480 per minute. In addition to this fixed mode of operation, the stimulator can also be used as a high current output stage for a programmable electrophysiologic stimulator.

\section{Animal Studies}

In vitro and in vivo experiments were performed in 55 dogs in order to assess the extent of burn injury to the esophagus caused by pacing with the pill electrode. These experiments conform to the principles of the American Physiological Society for experimental animal studies. In the in vitro series (25 dogs), the esophagus was excised, the electrode was inserted, and stimulation was performed for periods of 20 to 240 minutes with a rate of 60 to 120 beats per minute, pulse duration of $2 \mathrm{~ms}$, and current of 20 to $120 \mathrm{~mA}$. Multiple stimulations were performed using each esophageal specimen. After stimulating, the esophagus was examined for gross evidence of burning or ulceration. In the in vivo series (30 dogs), the animals were anes- 
thetized with $30 \mathrm{mg} / \mathrm{kg}$ of Nembutal and respired with room air. The esophagus was exposed via an approach medial to the common carotid artery and a suture was placed on the muscularis externa of the esophagus for later location of the point of stimulation. With the aid of a catheter as a guide, the pill electrode was inserted through the mouth into the esophagus to a point under the suture, as verified by palpation. The wire was then taped to the snout to prevent peristalsis from moving the electrode, and care was taken to insure that the esophagus was in its natural position relative to the other tissues of the neck.

Prior to stimulation, a muscle relaxant (Syncurine or Flaxedil) was administered and supplemented as necessary during stimulation to prevent skeletal muscle contractions from moving the electrode (the agents had no visible effect upon the contraction of the esophagus). The esophagus was stimulated for 10,30 , or 240 minutes at 60 or 120 pulses/min with a $2 \mathrm{~ms}$ pulse duration and current of 20,60 , or $80 \mathrm{~mA}$. After stimulation, the position of the electrode relative to the suture was again verified by palpation. Ten of the dogs were sacrificed immediately, while in each of the other 20 dogs, the wound was closed and the animal was allowed 1 to 3 days to recover so that inflammatory responses could develop and the extent of natural healing could be assessed.

When the esophagus was removed, it was rinsed of mucus, photographed, and examined grossly. In 25 of 30 in vivo experiments, the esophagus was then opened, rinsed of mucus, placed on filter paper, photographed, and fixed in buffered formalin. Following fixation for at least 24 hours, sections were taken of any lesions noted, with a diagram indicating the location of each lesion and the corresponding section. At least one section of apparently normal esophagus was also taken. In the event no lesion was evident on gross examination or with the aid of a hand lens, the entire circumference of the esophagus at the level of the electrode was taken (four to five strips of wall at 2 to 3 millimeters in thickness were embedded in paraffin block) and the entire area examined microscopically.

\section{Clinical Studies}

Tables I and II list the 46 subjects in whom we attempted transesophageal atrial pacing with the pill electrode or, in the nine children not old enough to swallow a capsule, with a catheter electrode introduced through the nose. This procedure was approved by our institutional review board and informed consent was obtained. Once initiated, pacing was continued for a period sufficient to perform either an electrophysiologic (EP) protocol or an imaging study. The EP studies consisted of determination of strength-duration curves for pacing thresholds, measurement of sinus node recovery time, and/or initiation and termination of supraventricular tachycardia. The imaging studies included radionuclide visualization of ventricular emptying sequences during normal and anomalous pathway activation, radionuclide ventriculography during pacing-induced stress, and/or echocardiography during pacing-induced stress.

\section{Electrophysiologic Applications}

In ten subjects, detailed strength-duration curves were obtained by noting the minimum current at which sustained capture was obtained at $2,4,6,8$, and $10 \mathrm{~ms}$ pulse duration. Current at which moderate patient discomfort occurred was also noted. In four subjects, sinus node recovery time was measured following standard electrophysiologic procedure; after 60 seconds of pacing at rates from 90 to 150 beats per minute, the pacing was terminated abruptly and the interval was measured between the last paced beat and the subsequent sinus escape beat.

Ten children with sustained reentrant supraventricular tachycardia, aged 1 week to 16 years, were treated with transesophageal overdrive atrial pacing on 16 different occasions. In the 16year-old patient, the pill electrode was used; in the others, nasogastric intubation was required. The three children under 1 year of age were sedated with chloral hydrate and slept through the procedure; one 2-year-old child (the first patient in this study) received general anesthesia; the remaining patients were either unsedated (1 patient) or received Valium ( $\leqslant 5 \mathrm{mg} \mathrm{IV}$ ) for sedation. Atrial flutter was present among five of the patients and recurrent supraventricular tachycardia in the other five. Transesophageal pacing was used to initiate as well as terminate the tachyarrhythmia in one of these five patients. Initial current strength and pulse duration were $4 \mathrm{~mA}$ and $4 \mathrm{~ms}$, respectively. Pulse duration was 
Table I.

Tachycardia Conversion

\begin{tabular}{|c|c|c|c|c|c|c|c|c|}
\hline $\begin{array}{c}\text { Episode/ } \\
\text { Patient }\end{array}$ & Age & Sex & Dx & Study & $\begin{array}{c}\text { Current } \\
\text { Level } \\
\text { (mA) }\end{array}$ & $\begin{array}{c}\text { Pulse } \\
\text { Duration } \\
\text { (ms) }\end{array}$ & $\begin{array}{c}\text { Charge } \\
(\mu \mathrm{C})\end{array}$ & Conversion \\
\hline $1 / 1$ & 2 & $F$ & PSVT & TERM & 9 & 3 & 27 & + \\
\hline $2 / 2$ & 13 & $\mathrm{~F}$ & $\mathrm{AF}$ & TERM & 20 & 10 & 200 & + \\
\hline $3 / 2$ & 13 & $\mathrm{~F}$ & $\mathrm{AF}$ & TERM & 10 & 5 & 50 & + \\
\hline $4 / 3$ & 9 & $\mathrm{~F}$ & $\mathrm{AF}$ & TERM & 12 & 6 & 72 & + \\
\hline $5 / 3$ & 9 & $\mathrm{~F}$ & $\mathrm{AF}$ & TERM & 12 & 4 & 48 & + \\
\hline $6 / 3$ & 9.5 & $\mathrm{~F}$ & $\mathrm{AF}$ & TERM & 14 & 5 & 70 & + \\
\hline $7 / 4$ & 5 & $M$ & $\mathrm{AF}$ & TERM & 17 & 4 & 68 & + \\
\hline $8 / 5$ & 6 days & $M$ & PSVT & TERM & 18 & 5 & 90 & + \\
\hline $9 / 5$ & 7 days & $M$ & PSVT & TERM & 16 & 5 & 80 & + \\
\hline $10 / 6$ & 14 & $M$ & PSVT & TERM & 18 & 10 & 180 & + \\
\hline $11 / 7$ & 8 weeks & $M$ & $\mathrm{AF}$ & TERM & 14 & 7 & 98 & + \\
\hline $12 / 8$ & 6 weeks & $\mathrm{F}$ & PSVT & TERM & 18 & 10 & 180 & NA \\
\hline $13 / 9$ & 16 & $\mathrm{~F}$ & $\mathrm{AF}$ & TERM & 12 & 5 & 60 & + \\
\hline $14 / 3$ & 9.5 & $\mathrm{~F}$ & $\mathrm{AF}$ & TERM & 16 & 6.5 & 104 & + \\
\hline $15 / 10$ & 6 weeks & $\mathrm{F}$ & WPW/SVT & TERM & 8 & 4 & 32 & + \\
\hline $16 / 10$ & 6 weeks & $\mathrm{F}$ & WPW/SVT & TERM & 12 & 5 & 60 & + \\
\hline $17 / 11$ & 24 & $M$ & Normal & $S / D$ & $\cdot$ & * & $*$ & NA \\
\hline $18 / 12$ & 32 & $M$ & Normal & S/D & * & * & * & NA \\
\hline $19 / 13$ & 50 & $M$ & Normal & S/D & * & * & * & NA \\
\hline $20 / 14$ & 51 & $M$ & Normal & S/D & * & * & * & NA \\
\hline $21 / 15$ & 30 & $M$ & Normal & $S / D$ & * & * & * & NA \\
\hline $22 / 16$ & 30 & $M$ & Normal & S/D & * & * & * & NA \\
\hline $23 / 17$ & 50 & $M$ & Normal & $S / D$ & * & * & * & NA \\
\hline $24 / 18$ & 27 & $M$ & Normal & $S / D$ & * & * & * & NA \\
\hline $25 / 19$ & 32 & $M$ & Normal & S/D & * & * & * & NA \\
\hline $26 / 20$ & 32 & $M$ & Normal & $S / D$ & * & * & * & NA \\
\hline
\end{tabular}

PSVT/SVT = paroxysmal/supraventricular tachycardia; WPW $=$ Wolff-Parkinson-White syndrome; $\mathrm{AF}=$ atrial flutter; TERM = termination; S/D = strength-duration; $\mathrm{NA}=$ not applicable; $+=$ successful; * $=$ variable.

increased by a $1 \mathrm{~ms}$ increment and current by 1 $\mathrm{mA}$ to a maximum of $10 \mathrm{~ms}$ and $20 \mathrm{~mA}$, respectively, until atrial capture was achieved. Once capture was achieved, pacing for $10-30 \mathrm{~s}$ was begun at successively shorter (by $10 \mathrm{~ms}$ ) drive cycle lengths until the tachycardia was interrupted or atrial refractoriness was reached.

\section{Imaging Applications}

Radionuclide ventriculography was performed during transesophageal pacing in seven patients with conduction abnormalities, in 12 patients with suspected coronary artery disease, and in 5 normal control subjects. At pulse durations of 3$15 \mathrm{~ms}$, current was increased until stable atrial capture occurred with a constant 1:1 stimulusto-QRS relationship at a rate $20-30$ beats faster than the sinus rate. This was continued for at least 3 minutes and, for the coronary artery disease group, was repeated at faster heart rates $(120,140,160$ beats per minute) until completion of the final stage of stress or occurrence of chest pain. In the conduction abnormality group, the images acquired during pacing were subjected to phase analysis ${ }^{18}$ in order to study the emptying sequence of the heart during normal and accessory pathway conduction. In the coronary artery disease group, images were obtained to determine the effect of pacing rate on ejection fraction, regional wall motion, relative ventricular volumes, and indices of diastolic filling (first-third filling time, peak filling rate, time-to-peak filling rate). In two normal subjects, M-mode echocardiograms were recorded at 1-minute intervals as the esophageal pacing rate was increased in order to observe changes in ventricular wall thickening, percent fractional shortening, and rate of shortening. 
Table II.

Radionuclide Ventriculography and Echocardiography

\begin{tabular}{|c|c|c|c|c|c|c|c|c|}
\hline Patient & Age & Sex & Dx & Study & $\begin{array}{c}\text { Current } \\
\text { Level } \\
\text { (mA) }\end{array}$ & $\begin{array}{c}\text { Pulse } \\
\text { Duration } \\
\text { (ms) }\end{array}$ & $\begin{array}{c}\text { Charge } \\
(\mu \mathrm{C})\end{array}$ & $\begin{array}{l}\text { Sustained } \\
\text { Capture }\end{array}$ \\
\hline 1 & 35 & $M$ & Normal & RVG & 15 & 7 & 105 & + \\
\hline 2 & 49 & $\mathrm{~F}$ & Normal & RVG & 12 & 15 & 180 & + \\
\hline 3 & 33 & $M$ & Normal & RVG & 20 & 5 & 100 & + \\
\hline 4 & 33 & $M$ & Normal & RVG & 15 & 5 & 75 & + \\
\hline 5 & 35 & $M$ & Normal & RVG & 15 & 5 & 75 & - \\
\hline 6 & 28 & M & WPW & RVG & 16 & 6 & 96 & + \\
\hline 7 & 62 & $M$ & WPW & RVG & 22 & 6 & 132 & + \\
\hline 8 & 12 & $M$ & WPW/SVT & RVG & 16 & 6 & 96 & + \\
\hline 9 & 31 & $M$ & WPW/SVT & RVG & 23 & 8 & 184 & + \\
\hline 10 & 46 & $\mathrm{~F}$ & WPW & RVG & 18 & 8 & 144 & + \\
\hline 11 & 18 & $M$ & Conc/path & RVG & 15 & 6 & 90 & + \\
\hline 12 & 15 & $\mathrm{~F}$ & Conc/path & RVG & 20 & 8 & 160 & + \\
\hline 13 & & $M$ & CAD & RVG & 16 & 9 & 144 & + \\
\hline 14 & & $M$ & CAD & RVG & 25 & 10 & 250 & + \\
\hline 15 & 63 & $M$ & CAD & RVG & 20 & 9 & 180 & + \\
\hline 16 & 50 & $M$ & CAD & RVG & 15 & 9 & 135 & + \\
\hline 17 & 55 & $M$ & CAD & RVG & 18 & 10 & 180 & + \\
\hline 18 & 54 & $M$ & CAD & RVG & 25 & 10 & 250 & - \\
\hline 19 & 72 & $M$ & CAD & RVG & 18 & 10 & 180 & + \\
\hline 20 & 35 & M & CAD & RVG & 15 & 5 & 75 & + \\
\hline 21 & & $M$ & CAD & RVG & 35 & 5 & 175 & - \\
\hline 22 & & $M$ & CAD & RVG & 20 & 3 & 60 & + \\
\hline 23 & 45 & $M$ & CAD & RVG & 25 & 4 & 100 & + \\
\hline 24 & & $M$ & CAD & RVG & 30 & 3 & 90 & + \\
\hline 25 & 51 & $M$ & Normal & Echo & 16 & 7 & 112 & + \\
\hline 26 & 28 & $M$ & Normal & Echo & 18 & 6 & 108 & + \\
\hline
\end{tabular}

WPW = Wolff-Parkinson-White syndrome; SVT = supraventricular tachycardia; CAD = coronary artery disease; $\mathrm{RVG}=$ radionuclide ventriculography; Echo = echocardiography; Conc/path = concealed pathway.

\section{Results}

\section{Safety}

In our animal experiments, esophagus that was excised with the original mucus undisturbed and then stimulated in vitro for 20 minutes required more than $100 \mathrm{~mA}$ to produce a visible lesion; with longer stimulation times, lesions occurred at lower currents: 60 minutes required $75 \mathrm{~mA}$ and 240 minutes required $60 \mathrm{~mA}$ to produce lesions. In the first five in vivo experiments, gross examination of the esophagus showed that 100 $\mathrm{mA}$ is required to produce burn injury after 10 minutes of stimulation and this heals within 3 days. Histological examination in the remaining 25 in vivo studies demonstrated that epithelial erosion can occur after stimulation at $60 \mathrm{~mA}$ for 4 hours, but is repaired in 24 to 48 hours. In addition, an inflammatory response may develop within 24 hours that involves the submucosal mucus ducts. Perforation or stricture does not occur and appears unlikely to develop. When the $60 \mathrm{~mA}$ stimulation was limited to 30 minutes (10 dogs), there was no evidence of mucosal ulceration or necrosis, although local infiltrates of neutrophils were found in otherwise intact mucosa and submucosa. These neutrophilic infiltrates were observed in and around mucus ducts. This is an occasional finding in normal control animals and was observed in sections taken from grossly uninvolved areas distant from the site of stimulation in the experimental animals.

In our clinical experiments, 43 of the 46 subjects who consented to transesophageal pacing were successfully paced for a period sufficient to perform one or more of the studies described above. There were no reports of discomfort in swallowing or positioning the pill electrode, and 
high quality recordings were obtained in all cases without difficulty. The chief complaint during pacing was of a sensation of moderate to severe "pulsatile heartburn" that disappeared immediately upon cessation of pacing. There were no reports of post-stimulation discomfort or pain upon swallowing.

\section{Electrophysiologic Applications}

Figure 6 is the strength-duration curve that shows the average transesophageal current necessary to effect sustained capture at pulse durations of $2-10 \mathrm{~ms}$ in 10 adults paced via the pill electrode. In terms of the Lapicque formulation, the strength-duration curve can be approximated by $\mathrm{I}=12.24 \mathrm{~mA}(1+3.12 \mathrm{~ms} / \mathrm{t}), \rho$ of 0.992 with a chronaxie of $3.12 \mathrm{~ms}$ and a rheobase of 12.24
$\mathrm{mA}$. The total variation in current is also shown. The current level at which discomfort was reported ranged from 15 to $25 \mathrm{~mA}$ and appeared to be only slightly affected by pulse durations, with wider pulses usually but not always producing discomfort at lower currents. Sinus node recovery times measured in four adults are summarized in Table III.

The results for tachycardia termination in the ten children are given in Table I. Transesophageal pacing was successful in terminating supraventricular tachycardia during all 16 tachycardia episodes in the ten patients. Mean current level for capture was $14.2 \mathrm{~mA}$ (range $8-20 \mathrm{~mA}$ ), and mean pulse duration was $5.9 \mathrm{~ms}$ (range 3-10). Mean charge was $88.69 \mu \mathrm{C}$ (range 32 to $200 \mu \mathrm{C}$ ). Threshold parameters (current strength and pulse duration) were not related to age or size. The

\section{STRENGTH-DURATION CURVE}

\section{CURRENT ( $\mathrm{mA})$}

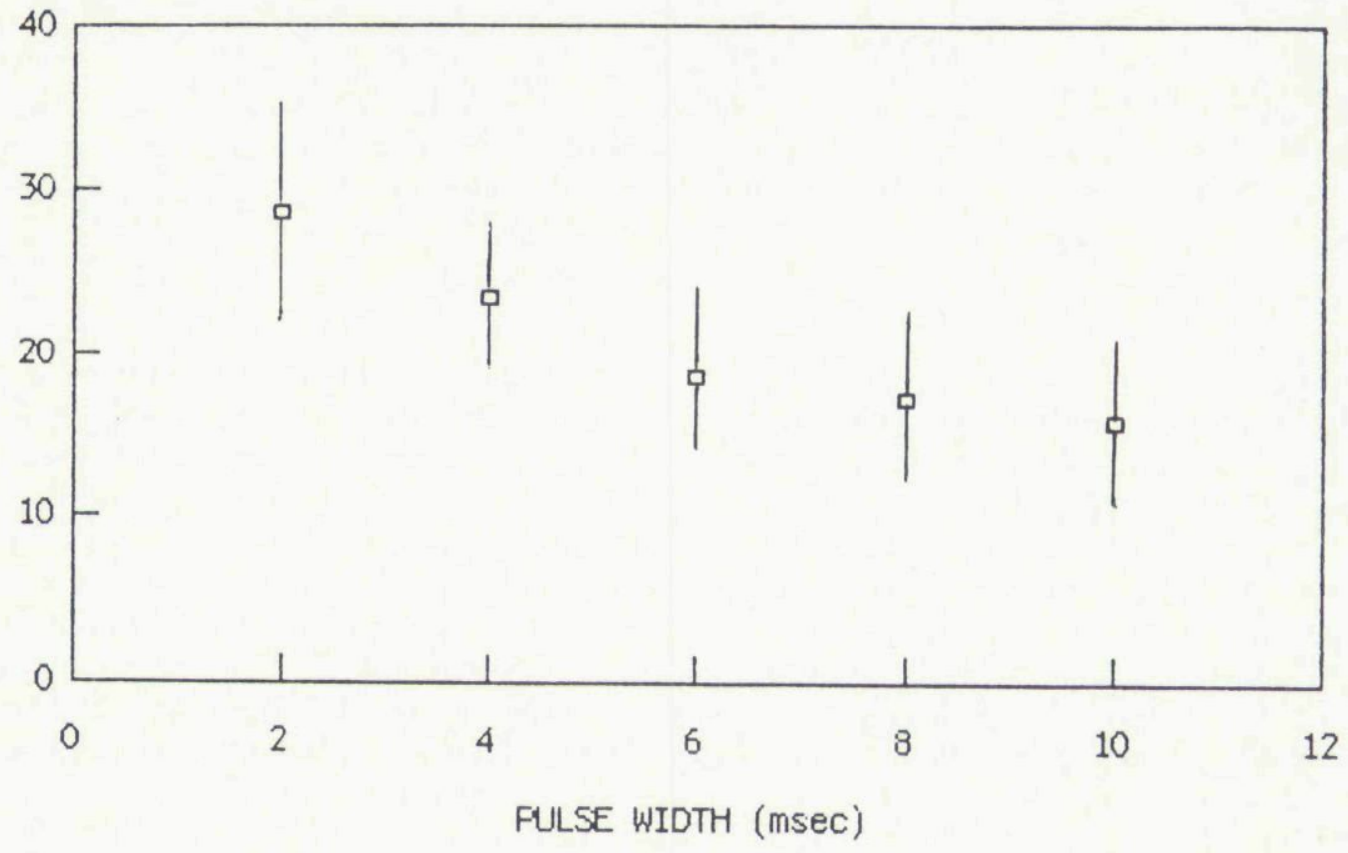

Figure 6. Mean strength-duration curve for ten normal subjects. The mean $( \pm S D)$ current level at which sustained capture was achieved at each of five selected pulse durations is plotted in this graph. 
Table III.

Sinus Node Recovery Time

\begin{tabular}{rcccc}
\hline & \multicolumn{5}{c}{ Subjects } \\
Heart rate & $\mathbf{1}$ & $\mathbf{2}$ & $\mathbf{3}$ & $\mathbf{4}$ \\
\hline 90 & 1000 & 1360 & - & 1000 \\
100 & 970 & - & - & - \\
110 & - & 1040 & 1080 & - \\
120 & - & 1200 & - & 1000 \\
130 & 960 & 1240 & 1070 & - \\
140 & - & - & - & 900 \\
150 & 920 & - & 900 & - \\
\hline
\end{tabular}

mean arrhythmia cycle length was $219 \mathrm{~ms}$ (range, $190-250 \mathrm{~ms}$ ). The mean pacing cycle length used during overdrive pacing to terminate the tachycardia was $170 \mathrm{~ms}(150-214 \mathrm{~ms})$. The mean pacing cycle length necessary to terminate the tachycardia was $78 \%$ of the mean tachycardia cycle length. Figure 7 shows supraventricular tachycardia which was converted by a train of transesophageal stimuli delivered for 3.8 seconds at a cycle length of $180 \mathrm{~ms}$. The top channels are leads I and II and the third channel is an esophageal electrogram. Upon cessation of pacing, the tachycardia converts to sinus rhythm. The only complication encountered in our studies was passage of the catheter into the trachea inducing paroxysms of coughing in one patient; this problem was immediately identified and the catheter was withdrawn. It was then properly positioned within the esophagus and successful conversion was performed.

\section{Imaging Applications}

Table II summarizes the results of transesophageal atrial pacing during imaging procedures. The first 24 subjects underwent radionuclide ventriculography and subjects 25 and 26 were imaged by echocardiography. Patients 6 to 12 were the subject of an earlier report ${ }^{18}$ that described the use of phase analysis for imaging. However, the detailed transesophageal pacing data have not been presented previously. In the four patients with electrophysiologically documented accessory pathways, transesophageal pacing induced a supraventricular tachycardia and therefore could be discontinued during radionuclide ventriculography, while in the remaining three patients and in four of the five control subjects, pacing was maintained throughout the image acquisition period. Stable pacing could not be achieved in the

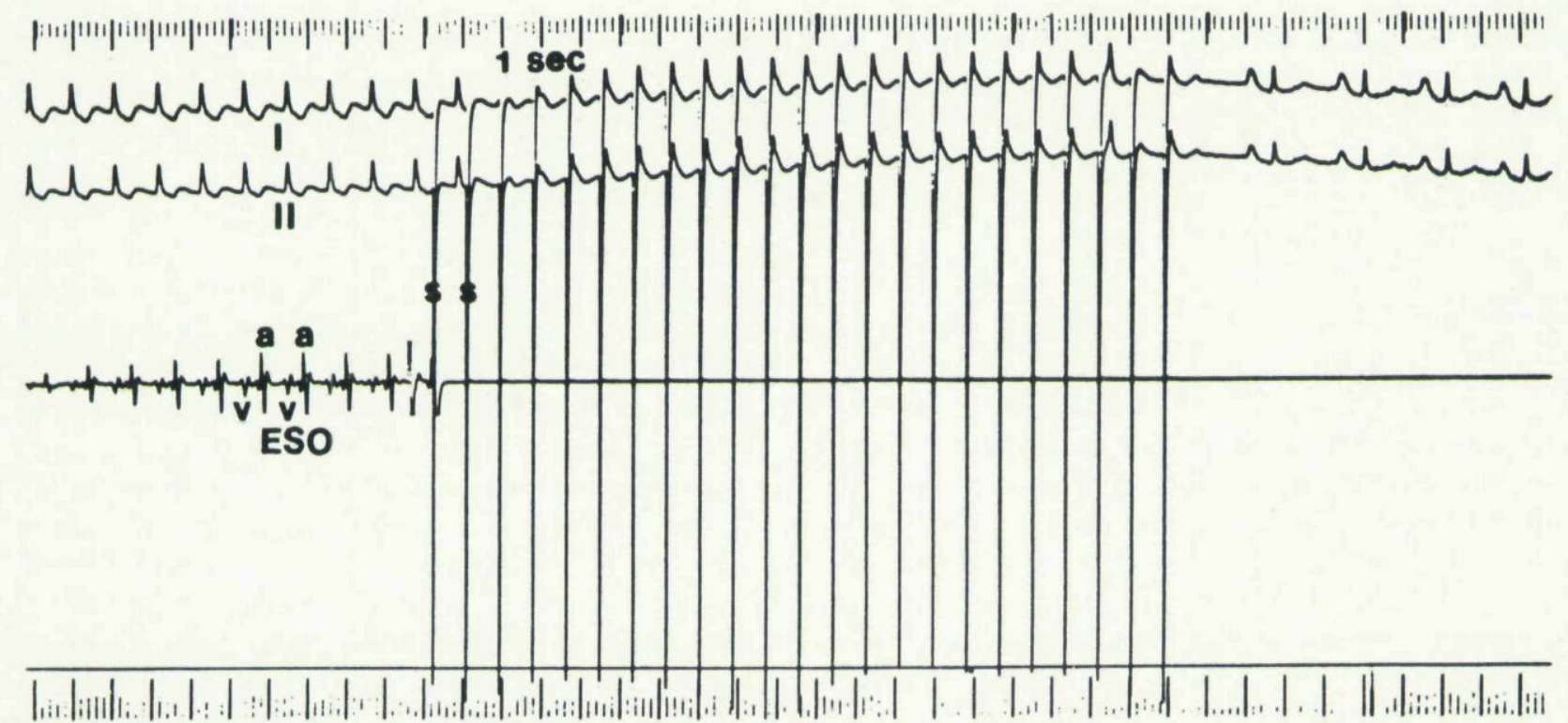

Figure 7. Transesophageal (ESO) atrial overdrive pacing in an infant with paroxysmal supraventricular tachycardia (SVT). The left-hand side of the figure demonstrates SVT with recording of both a transesophageal atrial (a) and a ventricular (v) electrogram. A train of transesophageal stimuli (s) were delivered for 3.8 seconds at a cycle length of $180 \mathrm{~ms}$; at termination of pacing, sinus rhythm appeared (right-hand side of figure). 
remaining subject at current levels he would tolerate. In the five normal subjects and the four subjects in whom supraventricular tachycardia was induced, phase analysis of images obtained during pacing revealed a similar pattern of emptying to that obtained during sinus rhythm. Of the three patients in whom pacing was maintained and tachycardia was not induced (all had Wolff-Parkinson-White syndrome), phase maps were obtained that localized the site of preexcitation and concealed pathways. For the seven subjects in this study in whom transesophageal pacing was maintained, mean current level was 19.3 $\mathrm{mA}$ (range, 12-23 $\mathrm{mA}$ ) and pulse duration $7.8 \mathrm{~ms}$ (range, 5-15 ms). Mean charge was 128.9 $\mu \mathrm{C}$ (range, 90-184 $\mu \mathrm{C}$ ).

In ten of the 12 coronary artery disease patients in whom radionuclide ventriculograms were used to examine ventricular function, transesophageal atrial pacing via the pill electrode was successful in providing stress without patient movement and high quality images were obtained. Target heart rates for all four stages were achieved in eight of ten patients. Mean charge was $139.4 \mu \mathrm{C}$ (range, 60-250 $\mu \mathrm{C}$ ). Mean current level was 20.2 $\mathrm{mA}$ (range, 15-30 $\mathrm{mA}$ ) and pulse duration was $7.25 \mathrm{~ms}$ (range, 3-10 ms). Similarly, elevated heart rate was easy to establish in echocardiographic imaging in two subjects, resulting in excellent M-mode quality. Mean current level was $17 \mathrm{~mA}$ and pulse duration was $6.5 \mathrm{~ms}$ with a mean charge of $110 \mu \mathrm{C}$. For all subjects in Table II for whom capture was sustained, the mean charge was $129.4 \mu \mathrm{C} \pm 46.9$.

\section{Discussion}

Two of our theoretical conclusions have been substantiated by our clinical results: that currents of about $25 \mathrm{~mA}$ are effective and that the very close bipolar spacing of the pill electrode does not compromise efficacy. The first of these conclusions is in agreement with recent investigations. ${ }^{12-17}$ However, the second conclusion appears to be contrary to the position of Gallagher ${ }^{14}$ who concluded that $30 \mathrm{~mm}$ was the optimal spacing in seven patients, but agrees with the most recent work of Benson, ${ }^{24}$ who found no significant difference between 15,22 , and $28 \mathrm{~mm}$ in his 30 patients. Our theoretical study suggests that the results found by others who used widely spaced electrodes represent a positioning of the electrode with one pole adjacent to the atrium and thus the pacing was essentially unipolar. The question of interelectrode spacing is an important one because close spacing protects against unintended stimulation of the ventricle, diaphragm, and chest and because it allows pharmaceutical encapsulation, thus eliminating the discomfort of intubation which, as Benson notes, ${ }^{16}$ is more objectionable than the discomfort of stimulation.

It should be pointed out again that careful electrocardiographic positioning is a firm requirement of close bipolar transesophageal pacing and that proper depth of insertion cannot be achieved by using markings on the wire (or catheter) together with radiographic or physical estimates of left atrial location. Even in individual patients, we have found differences in optimal insertion depth due to simple variations in seated posture, and even larger differences when a supine position is assumed. (We have occasionally observed that higher currents may be necessary when the patient is in a supine position even if the electrode appears to be optimally located electrocardiographically.) Andersen and Pless ${ }^{25}$ have recently recommended positioning at a fixed distance above the gastroesophageal junction in all patients, using a balloon distal to the electrodes for location and fixation at the gastric sphincter. We are not in agreement with this recommendation and prefer precise electrocardiographic positioning in all cases.

\section{Risk of Esophageal Injury}

We have concluded from our animal experiments that in short-term pacing (under $30 \mathrm{~min}$ utes) with pulse durations of $2 \mathrm{~ms}, 75$ to $100 \mathrm{~mA}$ will produce lesions, which appear to heal in 3 days. At longer pacing times (4 hours), $60 \mathrm{~mA}$ will produce similar epithelial erosions as well as inflammation involving the submucosal ducts. In the absence of mucosal destruction or ulceration, morbidity from these lesions seems unlikely. It appears clear that there is little or no risk of burn injury in short-term, low-current pacing (under one-half hour, less than $40 \mathrm{~mA}$ ) at pulse durations less than or equal to $2 \mathrm{~ms}$, but pacing for more than 4 hours may involve some risk. Also, pulse durations greater than 2 ms have not been studied and so these limits of current and pacing time should be lower if $5-10 \mathrm{~ms}$ pulses are used. 
One of the normal subjects included in this study consented to endoscopy before and on the first day after esophageal pacing and a second subject on the first day after. No evidence of coagulation necrosis was observed. Unfortunately, the discomfort associated with endoscopy was enormously greater than that associated with pill electrode swallowing and pacing, so that consent for an endoscopic procedure was not requested of our other subjects. In a 1972 study of transesophageal pacing in 92 patients, ${ }^{5}$ endoscopy was performed in 18. The only esophageal injuries noted were small decubital lesions in two patients, one of whom had been paced for 30 hours. These were interpreted as pressure necroses due to the nasogastric catheter used for pacing, rather than coagulation necroses. The patient in sinus arrest who was paced by Burack and Furman ${ }^{2}$ for 36 hours showed, at autopsy, only pressure necrosis caused by the nasogastric tube. Our animal results also suggest that short-term (onehalf hour) pacing at currents less than $40 \mathrm{~mA}$ at pulse durations less than $2 \mathrm{~ms}$ is safe.

We are beginning to adopt the intuitively appealing position that the use of increased pulse duration to reduce current results in the least patient discomfort although this opinion has not been confirmed by systematic study. This may increase the risk of esophageal injury and our dog studies performed with $2 \mathrm{~ms}$ stimuli should be repeated for longer pulse durations. With this slight reservation, it appears that temporary transesophageal pacing during the brief procedures described here is quite free of risk of esophageal burning. On the other hand, the safety of the technique for long-term support of a patient in sinus arrest, prior to permanent pacemaker implantation, cannot be presumed on the basis of our studies.

With respect to patient discomfort, the chief sensation elicited was an intermittent "heartburn" that was well tolerated for the length of the procedure. Although no subject described the sensation as painful, some were apprehensive and uncomfortable. No patient suffered ill effects from electrical stimulation of the esophagus and we are not aware of any short-term or longterm complications. Nevertheless, the technique should be attempted only with equipment that allows careful control of current levels and ensures patient isolation from line voltages.

\section{Applications}

The theoretical and experimental evidence of reentry as the mechanism of many forms of supraventricular tachycardia, including atrial flutter, has been convincingly demonstrated, ${ }^{26-28}$ and has resulted in a renewed interest in the role of overdrive pacing to terminate these arrhythmias. ${ }^{29-31}$ Overdrive pacing through either intracardiac, transthoracic, or transesophageal routes has been widely used in many clinical situations, ${ }^{14,16,30-32}$ and is especially important in the development of antiarrhythmia pacemakers as well as proper selection of candidates for these implanted devices. Transesophageal overdrive pacing offers several advantages: the technique offers virtually universal noninvasive access to the atrium; it can be performed in an outpatient setting; it requires minimal or no sedation; and there is little discomfort. It is important to note that three additional patients, not included in the tachycardia conversion group, were diagnosed by surface electrocardiogram as having atrial flutter; however, atrial recordings from the esophageal electrodes demonstrated atrial fibrillation. Therefore inappropriate overdrive pacing was not attempted.

Our experience with the use of transesophageal atrial pacing for initiation and termination of supraventricular tachycardia has been in children in whom venous catheterization is difficult, costly, and time consuming, particularly when repeated studies are done and appropriate vessels large enough to catheterize cannot be easily or safely accessed. Thus, our successful (10 of 10) use of the transesophageal route, like Benson's ${ }^{16,17,24}$ promises a bright future for the technique. In adults, the ability to use the pill electrode instead of a nasogastric tube provides additional advantage to the transesophageal route. Intracardiac pacing techniques will remain the definitive approach to arrhythmia and conduction disturbance elucidation; however, there is much appeal for a noninvasive alternative, particularly for sequential studies involved in serial drug testing.

A simple, noninvasive procedure using esophageal pacing coupled to radionuclide ventriculography had been shown to be of value in determining the site of pre-excitation in patients with Wolff-Parkinson-White syndrome and concealed accessory pathways. ${ }^{18}$ Scintigraphic phase maps 
agreed with electrophysiologic maps in localizing the pre-excited sites and concealed pathways. The technique may prove to be a valuable prelude to electrophysiology studies in patients who are surgical candidates for ablation of the bypass tract. The use of intracardiac atrial pacing to provide stress in the evaluation of patients with coronary artery disease has been extensively investigated using radiographic procedures in the catheterization laboratory. ${ }^{33-35}$ The radionuclide ventriculogram is ideally suited to provide many of the same diagnostic measurements of ventricular function, ${ }^{36-38}$ and thus the use of esophageal pacing during either radionuclide ventriculography or echocardiography provides a substitute for physiological stress, exercise, and the invasive risks of catheterization. The method allows complete control of heart rate independent of patient cooperation. Furthermore, the constant RR interval and resulting uniform diastolic filling provide images superior even to those obtained during rest. Moreover, it is applicable in patients who are unable to achieve intense levels of exercise,

\section{References}

1. Shafiroff, D.G.P., Linder, J.: Effects of external electrical pacemaker stimuli on the human heart. J. Thorac. Surg., 33:544, 1957.

2. Burack, B., Furman, S.: Transesophageal cardial pacing. Am. J. Cardiol., 23:469, 1969.

3. Rowe, G., Terry, W., Neblett, I.: Cardiac pacing with an esophageal electrode. Am. J. Cardiol., 24: $548,1969$.

4. Lubell, D.L.: Cardiac pacing from the esophagus. Am. J. Cardiol., 27:641, 1971.

5. Angel, J., Montoyo, J.F., et al.: Transesophageal electrical atrial stimulation. Rev. Esp. Cardiol., 25:525, 1972.

6. Roth, F., Satsmann, C., Gurtner, H.P.: Electrical stimulation of the heart via the esophagus. Schweiz. Med. Wochenschr., 99:164, 1969.

7. Meisner, H., Paek, S., Schober, R., et al.: Experience with an electrical conductive rubber electrode for esophageal pacing in infants. In Y. Watanabe (Ed.): Cardiac Pacing. Amsterdam, Excerpta Medica, 1977, p. 493.

8. Fields, J., Schachtman, J., Craig, S.D., et al.: Special electrode device for transesophageal cardioversion. Proc. 7th Int. Conf. on Medical and Biological Engineering, Stockholm, 1967.

9. Montoyo, J.V., Angel, J., Valle, V., et al.: Cardioversion of tachycardias by transesophageal atrial pacing. Am. J. Cardiol., 32:85, 1973.

10. Mitsui, T., Tanaka, T., Saijusa, M.: An esophageal balloon electrode for cardiac pacing. In H.J.Th. including patients with symptomatic claudication, unstable angina pectoris, chronic obstructive pulmonary disease, and patients taking drugs that produce $\beta$-blockade.

Several important aspects of transesophageal pacing have been presented, particularly the underlying theoretical principles and results of animal studies to assess the risk of esophageal injury. These give insight into the relationship of interelectrode distance, current level, and pulse duration to the achievement of successful capture with minimal risk and discomfort to the patient. Several new clinical applications have been described and preliminary results are discussed. The use of a miniature pill electrode encased in a pharmaceutical capsule obviates the need for bulky nasogastric tubes or intracardiac catheters and reduces many of these applications to outpatient procedures. Termination of tachycardia, basic electrophysiologic measurements, and controlled acceleration of heart rate represent procedures that can be performed noninvasively with this technique.

Thalen (Ed.): Cardiac Pacing. Assen, The Netherlands, 1973, p. 292. Van Gorcum.

11. Hartzler, G.O., Maloney, J.D.: Transesophageal atrial pacing in the Wolff-Parkinson-White Syndrome. Mayo Clin. Proc., 52:576, 1977.

12. Santini, M., Dini, F., Milazzotto, F., et al.: Transesophageal atrial stimulation in assessment of the recovery time of the sinus node. Minerva Cardioangiol., 25:111, 1977.

13. Sterz, H., Prager, H., Koller, H.: Transesophageal rapid stimulation of the left atrium in atrial tachycardias. Z. Kardiol., 67:3, 1977.

14. Gallagher, J.J., Smith, W.M., Kerr, C.R., et al.: Esophageal pacing: A diagnostic and therapeutic tool. Circulation, 65:336, 1982.

15. Kerr, C.R., Gallagher, J.J., Smith, W.M., et al.: The induction of atrial flutter and fibrillation and the termination of atrial flutter by esophageal pacing. PACE, 6:60, 1983.

16. Benson, D.W., Dunnigan, A., Sterba, R., et al.: Atrial pacing from the esophagus in the diagnosis and management of tachycardia and palpitations. J. Pediatr., 102:40, 1983.

17. Benson, D.W., Dunnigan, A., Benditt, D.G., et al.: Transesophageal study of infant supraventricular tachycardia: Electrophysiologic characteristics. Am. J. Cardiol., 52:1002, 1983.

18. Chan, W.W.C., Kalff, V., Dick, M., et al.: Topography of preemptying ventricular segments in patients with Wolff-Parkinson-White syndrome 
using scintigraphic phase mapping and esophageal pacing. Circulation, 67:1139, 1983.

19. Arzbaecher, R., Collins, S., Brown, D.D.: Transesophageal recording and pacing with a new pill electrode. Circulation, 56:II-173, 1978 (Abstract).

20. Arzbaecher, R.C., Jenkins, J.M., Collins, S.M., et al.: Atrial electrical activity: The view from the esophagus. In Frontiers of Engineering in Health Care. IEEE Press, 1979, p. 314

21. Arzbaecher, R.: A pill electrode for the study of cardiac arrhythmia. Med. Instrum., 12:227, 1978.

22. Jenkins, J., Wu, D., Arzbaecher, R.: Computer diagnosis of supraventricular and ventricular arrhythmias: A new esophageal technique. Circulation, 60:997, 1979.

23. Lapicque, L.: Definition expérimentale de 1 éxcitabilité. Soc. Biol., 2:280, 1909.

24. Benson, D.W., Sanford, M., Dunnigan, A., et al.: Transesophageal atrial pacing threshold: Role of interelectrode spacing, pulse duration and catheter insertion depth. J. Am. Coll. Cardiol. (In press).

25. Anderson, H.R., Pless, P.: Transesophageal pacing. PACE, 6:674, 1983

26. Wellens, H.J.J., Durrer, D.: The role of an accessory atrioventricular pathway in reciprocal tachycardia: Observations in patients with and without the Wolff-Parkinson-White syndrome. Circulation, 52:58, 1975.

27. Boineau, J.P., Schuessler, R.B., Moone, C.R., et al.: Natural and evoked atrial flutter due to circus movement in dogs. Am. J. Cardiol., 45:1167, 1980.

28. Inoue, H., Matsuo, H., Katayanagi, K., et al.: Clinical and experimental studies of the effects of atrial extrastimulation and rapid pacing on atrial flutter cycle lengths. Am. J. Cardiol., 48: 623, 1981.

29. Waldo, A.L., Plumb, V.J., Arciniegas, J.G., et al.: Transient entrainment and interruption of atrioventricular bypass pathway type of paroxysmal atrial tachycardia: A model for understanding and identifying reentry arrhythmias. Circulation, 67:73, 1983.

30. Waldo, A.L., MacLean, W.A.H., Karp, R.G., et al.: Continuous rapid atrial pacing to control recurrent or sustained ventricular tachycardia following open heart surgery. Circulation, 54:245, 1976.

31. Watson, R.M., Josephson, M.E.: Atrial flutter. I. Electrophysiologic substrates and modes of initiation and termination. Am. J. Cardiol., 45:732, 1982.

32. Dick, M., Campbell, R.M., Jenkins, J.M.: Thresholds for transesophageal atrial pacing. Catheterization and Cardiovasc. Diagn. 10:507, 1984.

33. Sowton, G.E., Balcon, R., Cross, D., et al.: Measurement of the angina threshold using atrial pacing. Cardiovasc. Res., 40:301, 1969.

34. Parker, J.O., Chiong, M.A., West, R.O., et al.: Sequential alterations in myocardial lactate metabolism, ST segments and left ventricular function during angina induced by atrial pacing. Circulation, 40:113, 1969.

35. Pasternac, A., Gorlin, R., Sonnenblick, E.H., et al.: Abnormalities of ventricular motion induced by atrial pacing in coronary artery disease. Circulation, 45:1195, 1972.

36. Stone, P., Dymond, D., Elliott, A.P., et al.: Use of first pass radionuclide ventriculography in assessment of wall motion abnormalities induced by incremental atrial pacing in patients with coronary artery disease. Br. Heart J., 43:369, 1980.

37. Tzivoni, D., Weiss, A.T., Bahat, V., et al.: Multiple gated blood pool cardiac scan (MUGA) during right atrial pacing (RAP): A sensitive method to detect myocardial ischemia. Am. J. Cardiol., 45: 408, 1980 (Abstract).

38. Hecht, H.S., Chew, C.D., Burnam, M., et al.: Radionuclide ejection fraction and regional wall motion during atrial pacing in stable angina pectoris: Comparison with metabolic and hemodynamic parameters. Am. Heart J., 101:726, 1981. 
This document is a scanned copy of a printed document. No warranty is given about the accuracy of the copy. Users should refer to the original published version of the material. 